\title{
In vitro cellular uptake of evodiamine and rutaecarpine using a microemulsion
}

\author{
This article was published in the following Dove Press journal: \\ International Journal of Nanomedicine \\ 17 May 2012 \\ Number of times this article has been viewed
}

\section{Yong-Tai Zhang \\ Zhe-Bin Huang \\ Su-Juan Zhang \\ Ji-Hui Zhao \\ Zhi Wang \\ Ying Liu \\ Nian-Ping Feng}

Department of Pharmaceutics, Shanghai University of Traditional

Chinese Medicine, Shanghai,

The People's Republic of China
Correspondence: Nian-Ping Feng Department of Pharmaceutics, School of Pharmacy, Shanghai University of Traditional Chinese Medicine, I 200 Cailun Road, Zhangjiang Hi-Tech Park, Pudong New District, Shanghai 20I203, The People's Republic of China

Tel +862151322198

Fax +862151322198

Email npfeng@hotmail.com
Objective: To investigate the cellular uptake of evodiamine and rutaecarpine in a microemulsion in comparison with aqueous suspensions and tinctures.

Materials and methods: A microemulsion was prepared using the dropwise addition method. Mouse skin fibroblasts were cultured in vitro to investigate the optimal conditions for evodiamine and rutaecarpine uptake with different drug concentrations and administration times. Under optimal conditions, the cellular uptake of microemulsified drugs was assayed and compared to tinctures and aqueous suspensions. Rhodamine B labeling and laser scanning confocal microscopy (LSCM) were used to explore the distribution of fluorochrome transferred with the microemulsion in fibroblasts. Cellular morphology was also investigated, using optical microscopy to evaluate microemulsion-induced cellular toxicity.

Results: The maximum cellular drug uptake amounts were obtained with a $20 \%$ concentration $(\mathrm{v} / \mathrm{v})$ of microemulsion and an 8 hour administration time. Drug uptake by mouse skin fibroblasts was lowest when the drugs were loaded in microemulsion. After incubation with rhodamine B-labeled microemulsion for 8 hours, the highest fluorescence intensity was achieved, and the fluorochrome was primarily distributed in the cytochylema. No obvious cellular morphologic changes were observed with the administration of either the microemulsion or the aqueous suspension; for the tincture group, however, massive cellular necrocytosis was observed.

Conclusion: The lower cellular uptake with microemulsion may be due to the fact that most of the drug loaded in the microemulsion vehicle was transported via the intercellular space, while a small quantity of free drug (released from the vehicle) was ingested through transmembrane transport. Mouse skin fibroblasts rarely endocytosed evodiamine and rutaecarpine with a microemulsion as the vehicle. The microemulsion had no obvious effect on cellular morphology, suggesting there is little or no cellular toxicity associated with the administration of microemulsion on mouse skin fibroblasts.

Keywords: mouse skin fibroblasts, evodiamine, rutaecarpine, microemulsion, cellular uptake, in vitro

\section{Introduction}

Evodiamine (Evo) and rutaecarpine (Rut) are important bioactive alkaloids isolated from the Chinese medicinal herb Evodia rutaecarpa (Juss.) Benth. ${ }^{1-3}$ Evo and Rut have been reported to exhibit anti-inflammatory ${ }^{4}$ and anti-nociceptive ${ }^{5}$ effects. Both Evo and Rut have been found to strongly inhibit prostaglandin E2 synthesis from lipopolysaccharide-treated RAW 264.7 cells, and Evo has also been shown to inhibit cyclooxygenase- 2 induction and NF- $\kappa$ B activation. ${ }^{6-9}$ However, these alkaloids are insoluble in water, which limits their transdermal absorption. In order to address 
this problem, an oil-in-water $(\mathrm{o} / \mathrm{w})$ microemulsion was designed as a vehicle to increase the solubility of these compounds. The application of this novel microemulsion could markedly improve the transdermal absorption of Evo and Rut compared to that obtained using aqueous suspensions and tinctures. ${ }^{10}$

Microemulsions have received increasing attention in recent years. A microemulsion is defined as a dispersion consisting of oil, surfactant, cosurfactant, and an aqueous phase, which is an optically isotropic and thermodynamically stable liquid solution with a droplet diameter of 10-100 nm, and it can therefore serve as an effective nanomedicine vehicle. ${ }^{11}$ Microemulsion systems have several advantages over conventional formulations, such as ease of manufacturing, increased drug solubility, good thermodynamic stability, and enhanced drug permeation. ${ }^{12}$ Microemulsions provide a promising delivery system for either sustained or controlled drug release through transdermal administration, ${ }^{10}$ and these novel transdermal delivery systems have attained increasing significance in basic research. For example, microemulsions may affect the stratum corneum (SC) layer of the skin, leading to increased fluidity of the bilayer, as well as interruption of the aqueous channels in the SC. These effects may result in the enhanced transdermal permeation of drugs. ${ }^{13-15}$ However, very few studies have been performed on the transport mechanism underlying microemulsion permeation through the $\mathrm{SC}$ into the dermis.

In animal tissue, fibroblasts are the most common cells in connective tissue. ${ }^{16}$ Dermal fibroblasts have been used extensively for a wide range of cellular and molecular studies, ${ }^{17}$ such as those on the mechanisms underlying skin aging, ${ }^{18-20}$ restoration of dermal wounds, ${ }^{21}$ dermal radiation injury, ${ }^{22}$ and construction of tissue-engineered skins. ${ }^{23-25}$

The purpose of this study was to investigate the cellular uptake of evodiamine and rutaecarpine in a microemulsion, as compared to that in aqueous suspensions and tinctures. Mouse skin fibroblasts were cultured in vitro and the amount of cellular uptake was measured using different concentrations, different drug administration times, and different formulations (ie, microemulsion, tincture, and suspension). These results may aid in determining the fate of microemulsions during dermal penetration.

\section{Materials and methods \\ Cell line}

Mouse skin fibroblasts (NIH/3T3) were purchased from the Shanghai Institute of Biochemistry and Cell Biology (Shanghai, China).

\section{Materials}

Evo and Rut (98\% purity, as determined by high-performance liquid chromatography [HPLC]) were provided by Linuo Biotechnology Co, Ltd (Zhengzhou, China). Ethyl oleate was purchased from Shanghai Yunhong Chemical Preparation Auxiliary Technology Co, Ltd (Shanghai, China). Polyethylene glycol PEG-35 castor oil (Cremophor ${ }^{\circledR}$ EL) was obtained from BASF (Ludwigshafen, Germany). Rhodamine B was obtained from Sigma-Aldrich (St Louis, MO). High-glucose Dulbecco's Modified Eagle's Medium (DMEM/High, Fisher Scientific Worldwide [Shanghai] Co Ltd, Shanghai, China), $0.25 \%$ trypsin, $0.02 \%$ ethylenediaminetetraacetic acid (EDTA), fetal calf serum, and phosphate buffered solution (PBS) were obtained from Shanghai Usen Biotechnology (Shanghai, China). All other chemicals were obtained from Sinopharm Chemical Reagent Co Ltd (Shanghai, China) and were of HPLC or analytical grade.

\section{HPLC analysis of Evo and Rut}

The LC-2010 A HT Liquid Chromatograph system (Shimadzu Corporation, Kyoto, Japan) was used for the dialysate sample analysis to detect the presence of Evo and Rut and to determine their concentrations. The HPLC system consisted of a double-plunger pump (Shimadzu Corporation), an autosampler (Shimadzu Corporation), an online degasser (Shimadzu Corporation), a Diamonsil C18 reverse phase column $(5 \mu \mathrm{m}, 4.6 \mathrm{~mm}$ [inner diameter] $\times 25 \mathrm{~cm}$; Dikma Technologies, Inc, Beijing, China), a column oven (Shimadzu Corporation), an ultraviolet detector (Hamamatsu Photonics, Hamamatsu, Japan), and a recording integrator (Shimadzu Corporation). The mobile phase was acetonitrile:water (43:57, $\mathrm{v} / \mathrm{v})$ containing $0.04 \%(\mathrm{w} / \mathrm{v})$ sodium 1-octanesulfonate with a flow rate of $1 \mathrm{~mL} /$ minute. The column temperature was constant at $35^{\circ} \mathrm{C}$ and the detection wavelength was $225 \mathrm{~nm}$.

\section{Preparation of microemulsion}

The microemulsion used in this study contained $0.1 \%$ (w/w) Evo and $0.1 \%(\mathrm{w} / \mathrm{w})$ Rut and was prepared using Cremophor EL $(33.8 \%, \mathrm{w} / \mathrm{w})$ as the surfactant, PEG-400 $(11.2 \%, \mathrm{w} / \mathrm{w})$ as the cosurfactant, and ethyl oleate $(5.0 \%$, w/w) as the oil phase. Evo and Rut were added to the ethyl oleate and stirred until they completely dissolved. The solution was then added to the mixture of Cremophor EL and PEG-400 and then stirred. The mixture was placed on a magnetic stirrer (RCT B S25; IKA, Staufen, Germany), at $300 \mathrm{r} /$ minute, and purified water $(50 \%, \mathrm{w} / \mathrm{w})$ was added dropwise. During this process, the appearance of the mixture turned from opaque to limpid, and the final preparation 
appeared opalescent-white when seen through light - an important feature of microemulsions.

\section{Characterization of microemulsion}

The average droplet size of the microemulsion was measured using the NanoSight LM series ${ }^{26}$ (NanoSight Ltd, Amesbury, UK). The viscosity of the prepared microemulsions was measured using a DV-I + Digital Viscometer (Brookfield Engineering Laboratories Inc, Middleboro, MA), with the No 1 rotor set at $100 \mathrm{rpm}$. Both measurements were performed in triplicate, and the final values were expressed in terms of mean \pm standard deviation. The appearance of the microemulsion was observed using a transmission electron microscope (Philips/FEI Tecnai 12, Amsterdam, The Netherlands). The sample was prepared with negative staining: the copper net was supported by Formvar film on a stencil plate, the microemulsion was dropped gently on the film, and the film was allowed to dry for approximately 20 minutes. A drop of $0.5 \%$ uranyl acetate was then added to the film and allowed to dry for 10 minutes. The sample was then observed and the image was captured.

\section{Cell culture}

The cell line was removed from storage in liquid nitrogen and placed in a $37^{\circ} \mathrm{C}$ water bath for 1 minute. Then, $10 \mathrm{~mL}$ of fresh culture medium (DMEM/High) was added to the culture dish with a transfer pipette and it was mixed uniformly. The culture dish was placed in a $\mathrm{CO}_{2}$ incubator (Forma 3111; Thermo Fisher Scientific, Walther, MA) for 24 hours $\left(5 \% \mathrm{CO}_{2}\right.$, incubator at $\left.37^{\circ} \mathrm{C}\right)$. Subsequently, the culture medium was extracted using a vacuum pump; $2 \mathrm{~mL}$ PBS was added to clean the dish and was then removed by the vacuum pump. Zymine solution $(1 \mathrm{~mL})$ was added and mixed, and the excess zymine solution was poured out. The cells in the dish were digested at $37^{\circ} \mathrm{C}$ for 3 minutes, until they floated, and $4 \mathrm{~mL}$ of culture medium was used to clean the cells. The cell suspension was distributed into a new culture dish and $8 \mathrm{~mL}$ of fresh culture medium was added for subcultivation. Cultures were maintained at $37^{\circ} \mathrm{C}$ under humidified conditions with $5 \% \mathrm{CO}_{2}$. All procedures were performed on a super-clean bench (VCM-620; Dabao Instrument Company, Suzhou, China) and aseptic techniques were maintained.

\section{Preparation of formulations for comparison}

The aqueous suspension was prepared with pure water and the tincture with $70 \%(\mathrm{w} / \mathrm{w})$ ethanol. The concentrations of Evo $(0.1 \%, w / w)$ and Rut $(0.1 \%, w / w)$ in the aqueous suspension and the tincture were identical to those in the microemulsion.

\section{Cellular uptake of Evo and Rut in vitro}

Mouse skin fibroblasts in the log phase were transferred to a six-well plate culture dish at $5 \times 10^{5}$ cells/unit. An atmosphere of $5 \% \mathrm{CO}_{2}$ was maintained for 12 hours at $37^{\circ} \mathrm{C}$. The prepared formulations were injected into the holes, replacing equal volumes of culture fluid, at $37^{\circ} \mathrm{C}$ in an atmosphere of $5 \% \mathrm{CO}_{2}$. After incubation, the culture fluid was removed and the holes were washed three times with $1 \mathrm{~mL}$ of PBS and $0.5 \mathrm{~mL}$ of normal saline. The cells were then carefully scraped and transferred into centrifuge tubes that were placed in an icewater bath for ultrasonication with a ultrasonic cell disruptor (JY99-IIDN; LifeScientz Bio-tech Co Ltd, Ningbo, China) at $900 \mathrm{w}$ for 10 seconds. Acetic ether $(200 \mu \mathrm{L})$ was then added and the mixture was shaken carefully, followed by extraction of the supernatant liquid. This process was performed in triplicate and the supernatant liquids were combined. The combined supernatant liquid was volatilized through nitrogen using a pressured gas-blowing concentrator (Shanghai ANPEL Scientific Instrument Co Ltd, Shanghai, China) in a draught cupboard. After the liquid dried, $100 \mu \mathrm{L}$ methanol was added to dissolve the components using a vortex mixer (XW-80A; Shanghai Jingke Scientific Instrument Co Ltd, Shanghai, China), vibrated for 5 minutes, and analyzed with HPLC after centrifugation (5452; Eppendorf AG, Hamburg, Germany) at 10,000 r/minute for 5 minutes.

\section{Cellular uptake of fluorescence-labeled microemulsion}

Evo and Rut were replaced with rhodamine B methanol solution $(1.0 \mathrm{mg} / \mathrm{mL}: 10.0 \mathrm{mg}$ rhodamine $\mathrm{B}$ dissolved in $10 \mathrm{~mL}$ methanol), and the concentration of rhodamine $\mathrm{B}$ in the prepared microemulsion was $10 \mu \mathrm{g} / \mathrm{mL}$. Some volume of culture medium in the glass culture dish (used for LSCM) was pipetted and replenished with an equal volume of rhodamine B-labeled microemulsion solution. The cells were then incubated in a $\mathrm{CO}_{2}$ incubator, with an atmosphere of $5 \% \mathrm{CO}_{2}$ maintained for 8 hours at $37^{\circ} \mathrm{C}$. The culture fluid was then removed and the culture dish was washed 3 times with $1 \mathrm{~mL}$ PBS. The cells were optically scanned at different increments through the z-axis of an LSCM (TCS SP2; Leica Microsystems, Wetzlar, Germany). Optical excitations were performed with a $554 \mathrm{~nm}$ argon laser beam and fluorescence emission was detected at $575 \mathrm{~nm} .{ }^{27}$ 


\section{Results and discussion \\ Preparation and characterization of microemulsions}

With the dropwise addition of water, the mixture underwent a phase transition from gel to microemulsion. The microemulsion was diluted to $1000 \times(\mathrm{w} / \mathrm{w})$ and the mean droplet size was $74 \pm 7.07 \mathrm{~nm}$, as measured by NanoSight (Figure 1). The viscosity of the microemulsion was $37.65 \pm 0.14 \mathrm{mPa} \cdot \mathrm{s}$. The microemulsion showed adequate fluidity, which ensured free movement of the nanoparticles in the microemulsion fluid. The droplets were spherical and remained separate when observed by transmission electron microscopy (Figure 2).

\section{Cellular uptake of Evo and Rut in vitro}

Five concentrations of microemulsion liquid $(5 \%, 10 \%$, $15 \%, 20 \%$, and $30 \% ; \mathrm{v} / \mathrm{v}$ ) were evaluated in this study. The results showed that the amount of uptake increased with increases in the microemulsion concentration and began to taper after the microemulsion accounted for $20 \%$ of the culture fluid (Figure 3). Within a concentration range of $5 \%-20 \%$, the uptake amounts for Evo and Rut were dose dependent, indicating that cellular uptake may occur via passive transport. High microemulsion concentrations increased the concentration of the drugs in the culture fluid, but an excess of microemulsion components (eg, surfactant and cosurfactant) may hinder cellular uptake.

As a $20 \%$ microemulsion concentration represented uptake saturation, the effect of incubation time on the amount of cellular uptake was investigated at this concentration (Figure 4). The amount of cellular uptake increased with the time from 2 to 8 hours of incubation. Uptake for both drugs reached a maximum at 8 hours, changed little until 12 hours, and then decreased slightly.
A tincture and an aqueous suspension were used as comparative formulations for further study. Under the same conditions ( $20 \%$ concentration of each formulation, 8 hour incubation time), the results (Figure 5) showed that the uptake amounts for the microemulsion were the smallest and those for the aqueous suspension were the largest. Evo and Rut are small molecules and highly lipid soluble. When dispersed in water, they are likely to be absorbed by cells. However, high amounts of alcohol in the tincture caused massive cellular necrocytosis, which resulted in a considerably lower drug uptake in this group, as compared with the aqueous suspension group. The mechanisms of nanoparticle-cell interaction are still not completely understood. dos Santos et al indicated that the internalization of (nano)particles was highly size dependent for all the cell lines studied, and that (nano)particle uptake may not follow the commonly defined size limits for uptake processes..$^{28}$ Their results highlight the variability of uptake kinetics for one material in different cell types. ${ }^{28}$ In general, extracellular matrix proteins have a negative charge, and the particles with a positive charge can adhere to the cell membrane. On the contrary, particles with a positive charge may weaken the adhesivity of the cell. ${ }^{29,30}$ Other reports ${ }^{31,32}$ have shown that nanoparticles have a positive surface charge that can attract and combine nonspecific static from the negative charges of glycoprotein, proteoglycan, and phospholipid on the cell surface, and that this effect depends on the quantity of positive charges on the surface of the nanoparticle and on the negatively charged macromolecules from the cytomembrane. For example, liposomes have a positive surface charge that can increase the affinity between nanoparticles and cells. ${ }^{33-35}$ The microemulsion with the zeta potential $-1.02 \pm 0.18$ was expected to have a negative surface charge on the basis of the presented composition, which may have adversely affected the adhesivity of the cell, further
A

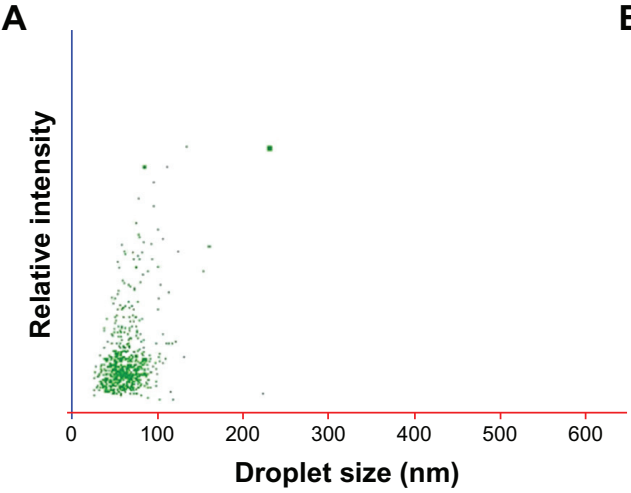

B



Figure I Microemulsion droplet size measured by NanoSight (NanoSight Ltd, Amesbury, UK). (A) Droplet size and relative intensity. (B) Still frame of a video of microemulsion droplets.

Note: The droplets appear as bluish-green spots (taken using NanoSight). 


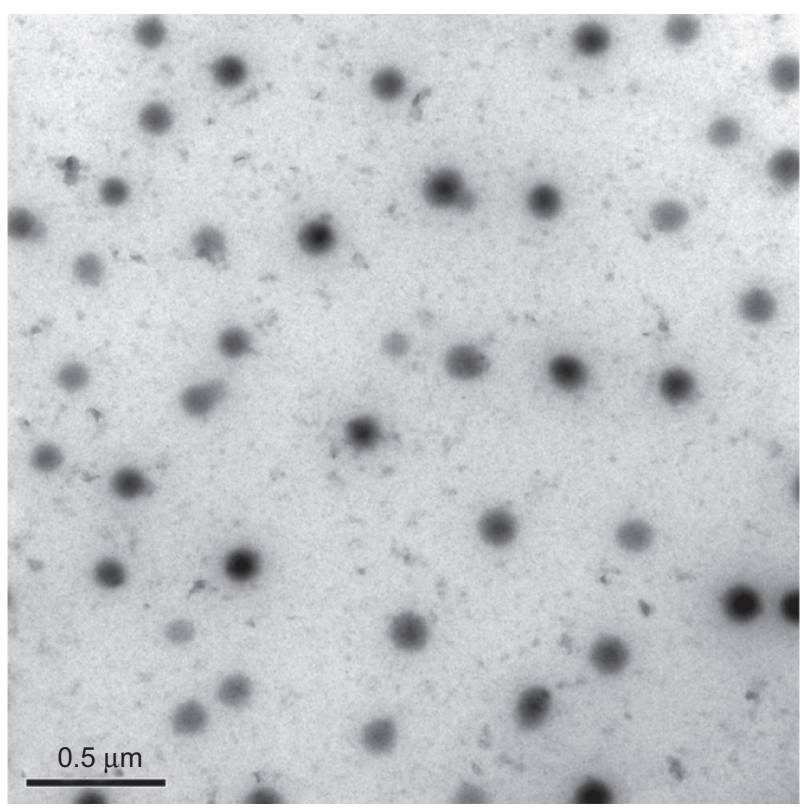

Figure 2 Transmission electron microscopy image of Evo- and Rut-loaded microemulsion (stained with uranyl acetate).

decreasing cellular uptake. Research has also shown that the incubation temperature can influence the cellular uptake of nanoparticles, as the uptake efficiency at $37^{\circ} \mathrm{C}$ is higher than that at $4^{\circ} \mathrm{C}$, which indicates that the uptake process is energy dependent. ${ }^{36}$ The microemulsion used in this study consisted of droplets with an average size of $74 \pm 7.07 \mathrm{~nm}$, but the amount of cellular uptake was lower than that with the aqueous suspension. It is possible that a large distribution of droplet sizes hindered cellular uptake (Table 1).

Previous studies have shown that microemulsions cause significantly enhanced Evo and Rut permeation through the skin, as compared to conventional preparations such as tincture and aqueous suspension. ${ }^{10}$ However, in the current study, the in vitro uptake of Evo and Rut in mouse skin fibroblasts was smaller for the microemulsion than



Figure 3 Effect of microemulsion concentration on the amount of Evo (blue) and Rut (red) uptake by mouse skin fibroblasts.

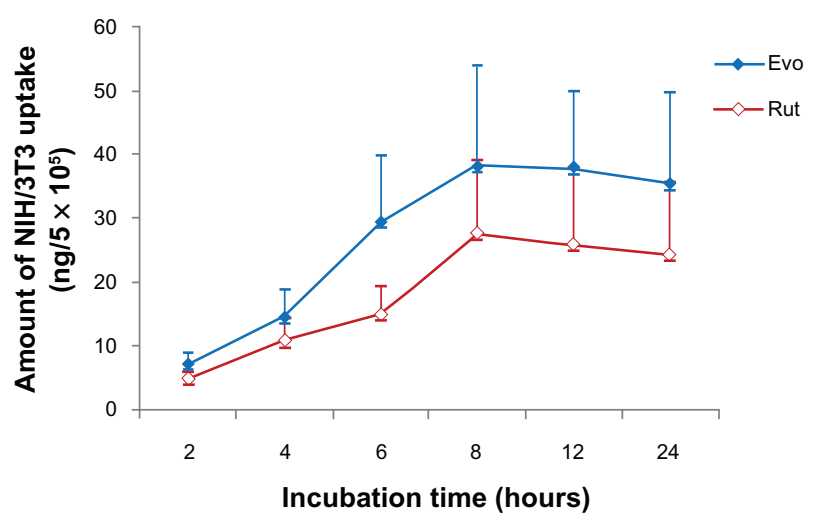

Figure 4 Effect of microemulsion incubation time on the amount of Evo and Rut uptake by mouse skin fibroblasts.

for the tincture and the aqueous suspension. These results suggest that most of the drugs loaded in a microemulsion are transported via intercellular spaces, while a small quantity of free drug may be released from the vehicle and ingested through transmembrane transport. In light of the results from previous in vivo studies, ${ }^{10}$ a possible route of microemulsion-enhanced percutaneous absorption is as follows: the microemulsion increases the fluidity of the bilayer and interrupts the aqueous channels in the SC, leading to ingestion by cells in the SC; the accumulated microemulsion then creates a high drug-concentration gradient, which causes diffusion into the dermal intercellular spaces, thereby enhancing transdermal drug permeation. ${ }^{37,38}$

\section{Influence of microemulsion on cellular appearance}

Various vehicles loaded with Evo and Rut affected cellular morphology (Figure 6). For all formulations, a concentration of $20 \%$ and an incubation time of 8 hours were used. Compared to normal cells, cells incubated with microemulsion (whether or not the microemulsion was loaded with drugs; Figures 6-8) showed no obvious changes. In addition, no

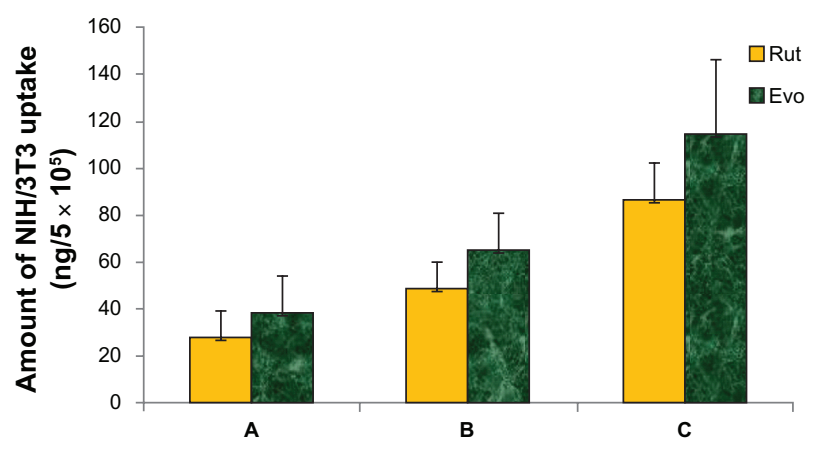

Figure 5 Effect of different formulations (A) microemulsion, (B) tincture, and (C) aqueous suspension, on the amount of Evo and Rut uptake by mouse skin fibroblasts. 
Table I Size distribution and droplet concentration statistics of microemulsion by NanoSight

\begin{tabular}{lll}
\hline $\begin{array}{l}\text { Droplet size } \\
(\mathbf{n m})\end{array}$ & $\begin{array}{l}\text { Droplet concentration } \\
\left(10^{6} / \mathrm{mL}\right)\end{array}$ & $\begin{array}{l}\text { Percent size } \\
\text { decrease }(\%)\end{array}$ \\
\hline 10 & 0 & 0 \\
30 & $46.652 \pm 1.703$ & $0.41 \pm 0.08$ \\
50 & $302.559 \pm 116.931$ & $14.70 \pm 7.30$ \\
70 & $377.189 \pm 25.052$ & $53.34 \pm 13.85$ \\
90 & $193.157 \pm 49.463$ & $82.02 \pm 8.71$ \\
110 & $59.806 \pm 24.223$ & $91.67 \pm 4.56$ \\
290 & $62.921 \pm 31.676$ & 100.00 \\
\hline
\end{tabular}

morphologic change was noted in cells incubated with the aqueous suspension (Figure 6). The drugs (Evo and Rut) had no apparent effect on the cells at the dosage used, as shown by the similar cellular morphology seen in cells incubated with the microemulsion vehicle without drugs (Figures 6-8). However, cells incubated with the tincture shrank sharply and underwent necrocytosis (Figure 6), as indicated by the amount of cellular debris observed floating in the culture fluid. This effect may have been due to the high alcohol content of the tincture.

In comparison with normal cellular morphology (Figure 7), it was found that microemulsion administration did not lead to transformation of the cellular morphology. Furthermore, the cellular morphology did not obviously transform with an increase in the microemulsion concentration. Pharmaceutical adjuncts of the prepared microemulsion, such as PEG-400 $(11.2 \%, \mathrm{w} / \mathrm{w})^{39}$ and Cremophor EL $(33.8 \%$, w/w), are used extensively in the pharmaceutical industry. Cremophor EL use has been reported, up to the $50 \%$ level, as a solubilizer

A



C

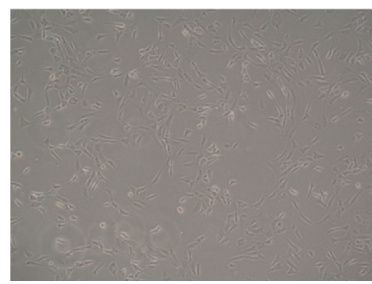

Figure 6 Influence of incubation with different formulations: (A) tincture; (B) aqueous suspension; (C) microemulsion; and (D) microemulsion without Evo and Rut on cellular morphology.



Normal



$15 \%$

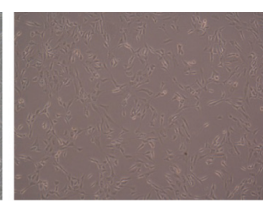

$5 \%$

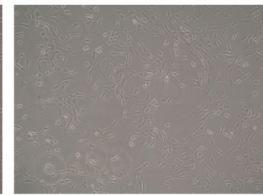

$20 \%$

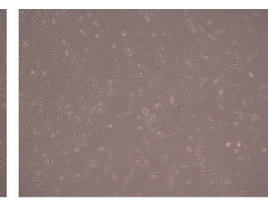

$10 \%$



$30 \%$
Figure 7 Influence of microemulsion concentration on cellular morphology.

in preparations of paclitaxel injections, ${ }^{40}$ and when used in this study, no obvious transformation of cellular morphology was observed with an inverted microscope. Ethyl oleate is extensively used in topical medications and is designated by the US Food and Drug Administration as harmless, which is supported by the results of a 91-day feeding study in rats. ${ }^{41}$ This evidence supports the finding that the microemulsion has little cellular toxicity.

\section{Cellular uptake of fluorescence-labeled microemulsion}

Using LSCM, orange fluorescence was observed in the cytoplasm of mouse skin fibroblasts, clearly showing that, after uptake, the fluorescein was distributed in the cytoplasm of these cells. The fluorescence increased with the microemulsion concentration, as well as with incubation time (Figures 9 and 10, respectively). Interestingly, a variation in the amount of orange fluorescence was seen in accordance with the uptake amounts of Evo and Rut, as determined by HPLC.

The fluorescence intensity of the microemulsion group was relatively lower than that of the tincture and aqueous suspension groups (Figure 11), which may be related to the lowest uptake amount of rhodamine B with microemulsion as



2 hours

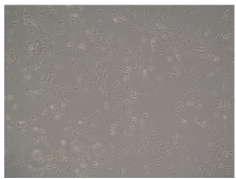

8 hours

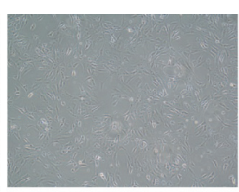

4 hours

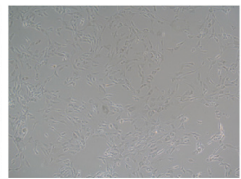

12 hours

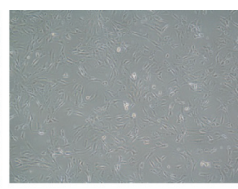

6 hours

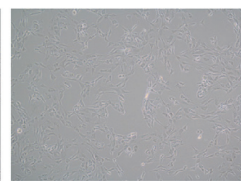

24 hours
Figure 8 Influence of microemulsion incubation time on cellular morphology. 




$5 \%$



$20 \%$

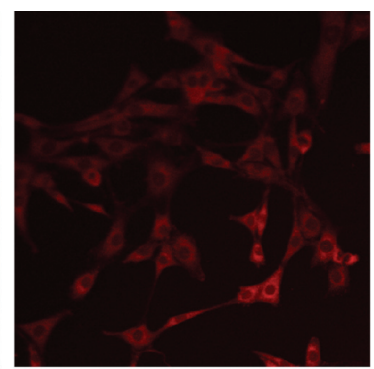

$10 \%$

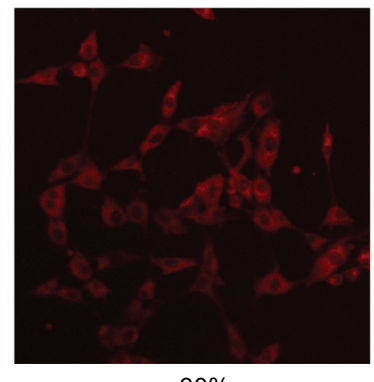

$30 \%$
Figure 9 LSCM images showing different concentrations of rhodamine B-labeled microemulsion incubated with mouse skin fibroblasts.

Abbreviation: LCSM, laser scanning confocal microscopy.

the vehicle. When the lipophilic rhodamine B was loaded in the microemulsion, it was difficult to release and, therefore, difficult for cells to ingest, while the free molecules of rhodamine B in the aqueous suspension were easily captured. Cells from the tincture group showed small, diffuse, and indistinct fluorescence, which may be due to the fact that the high alcohol content led to extensive cellular death and rupture, resulting in the leakage of fluorochrome out of the cells, staining the background.

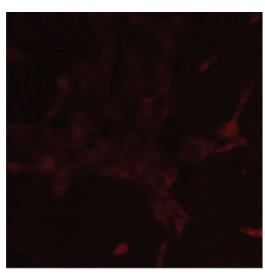

2 hours

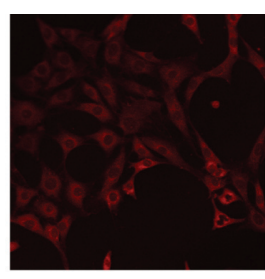

6 hours

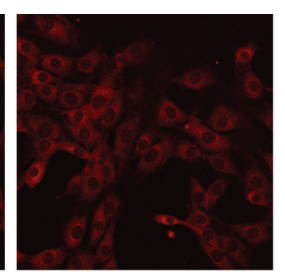

8 hours

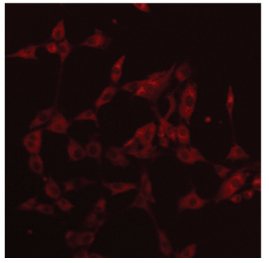

12 hours

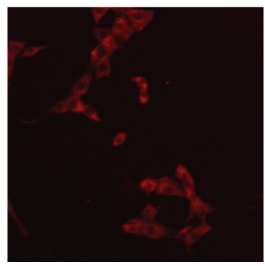

24 hours
Figure 10 LSCM images showing mouse skin fibroblasts incubated with rhodamine B-labeled microemulsion for different incubation times.

Abbreviation: LCSM, laser scanning confocal microscopy.
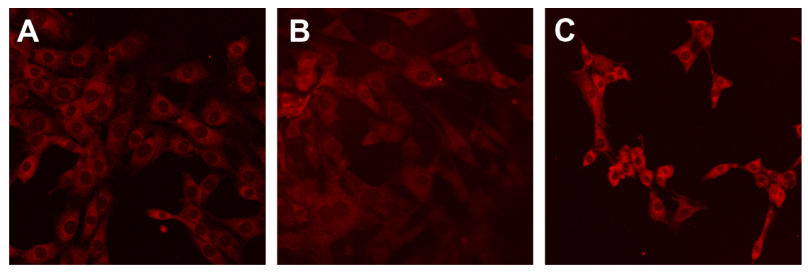

Figure I I LSCM images showing rhodamine B-labeled formulations (A) microemulsion; (B) tincture; and (C) aqueous suspension.

Abbreviation: LCSM, laser scanning confocal microscopy.

\section{Conclusion}

The amount of cellular uptake with microemulsion was relatively lower than with tincture and aqueous suspension, which may be due to the fact that most of the drug loaded in the microemulsion vehicle was transported via the intercellular space, while a small quantity of free drug (released from the vehicle) was ingested through transmembrane transport. The prepared microemulsion was harmless to mouse skin fibroblasts, while tincture administration led to obvious cellular toxicity. This research may provide the foundation and inspiration for understanding the absorption mechanism of microemulsion as a transdermal delivery system.

\section{Acknowledgments}

This work was financially supported by grants J50302 and 09JW10 from the Shanghai Education Committee, program 10XD14303900, from the Science and Technology Commission of Shanghai Municipality, and projects NCET08-0898 and IRT1071 from the State Education Ministry of the PR China.

The authors would like to thank Professor Wu-Xiong Zhou for taking LSCM photos and Zhong-Hua Wu for culturing cells.

\section{Disclosure}

The authors report no conflicts of interest in this work.

\section{References}

1. Yu PL, Chao HL, Wang SW, Wang PS. Effects of evodiamine and rutaecarpine on the secretion of corticosterone by zona fasciculata-reticularis cells in male rats. J Cell Biochem. 2009;108(2):469-475.

2. Zhao MY, Yang XW. Optimization of the extraction conditions and simultaneous quantification by RP-LC of six alkaloids in Evodiae fructus. Chromatographia. 2008;67(7-8):543-550.

3. Yang XW, Teng J. Chemical constituents of the unripe fruits of Evodia rutaecarpa. J Chin Pharm Sci. 2007;16(1):20-23.

4. Matsuda H, Yoshikawa M, Iinuma M, Kubo M. Antinociceptive and anti-inflammatory activities of limonin isolated from the fruits of Evodia rutaecarpa var bodinieri. Planta Med. 1998;64(4):339-342.

5. Kobayashi Y. The nociceptive and anti-nociceptive effects of evodiamine from fruits of Evodia rutaecarpa in mice. Planta Med. 2003;69(5): 425-428. 
6. Moon TC, Murakami M, Kudo I, et al. A new class of COX-2 inhibitor, rutaecarpine from Evodia rutaecarpa. Inflamm Res. 1999;48(12): $621-625$.

7. Yarosh DB, Galvin JW, Nay SL, Peña AV, Canning MT, Brown DA. Anti-inflammatory activity in skin by biomimetic of Evodia rutaecarpa extract from traditional Chinese medicine. J Dermatol Sci. 2006;42(1): 13-21.

8. Ko HC, Wang YH, Liou KT, et al. Anti-inflammatory effects and mechanisms of the ethanol extract of Evodia rutaecarpa and its bioactive components on neutrophils and microglial cells. Eur J Pharmacol. 2007;555(2-3):211-217.

9. Choi YH, Shin EM, Kim YS, Cai XF, Lee JJ, Kim HP. Anti-inflammatory principles from the fruits of Evodia rutaecarpa and their cellular action mechanisms. Arch Pharm Res. 2006;29(4):293-297.

10. Zhang YT, Zhao JH, Zhang SJ, et al. Enhanced transdermal delivery of evodiamine and rutaecarpine using microemulsion. Int J Nanomedicine. 2011;6:2469-2482.

11. Tenjarla S. Microemulsions: an overview and pharmaceutical applications. Crit Rev Ther Drug Carrier Syst. 1999;16(5):461-521.

12. Lawrence MJ, Rees GD. Microemulsion-based media as novel drug delivery systems. Adv Drug Deliv Rev. 2000;45(1):89-121.

13. Hathout RM, Mansour S, Mortada ND, Geneidi AS, Guy RH. Uptake of microemulsion components into the stratum corneum and their molecular effects on skin barrier function. Mol Pharm. 2010;7(4):1266-1273.

14. Dreher F, Walde P, Walther P, Wehrli E. Interaction of a lecithin microemulsion gel with human stratum corneum and its effect on transdermal transport. J Control Release. 1997;45(2):131-140.

15. Gloor M, Gehring W. Effects of emulsions on the stratum corneum barrier and hydration. Hautarzt. 2003;54(4):324-330. German.

16. Weissman-Shomer P, Fry M. Chick embryo fibroblasts senescence in vitro: pattern of cell division and life span as a function of cell density. Mech Ageing Dev. 1975;4(2):159-166.

17. Piérard GE, Piérard-Franchimont C. From cellular senescence to seven ways of skin aging. Rev Med Liege. 1997;52(4):285-288. French.

18. Gentile M, Latonen L, Laiho M. Cell cycle arrest and apoptosis provoked by UV radiation-induced DNA damage are transcriptionally highly divergent responses. Nucleic Acids Res. 2003;31(16):4779-4790.

19. Chung JH, Seo JY, Choi HR, et al. Modulation of skin collagen metabolism in aged and photoaged human skin in vivo. J Invest Dermatol. 2001;117(5):1218-1224.

20. Tsukahara K, Takema Y, Moriwaki S, et al. Selective inhibition of skin fibroblast elastase elicits a concentration-dependent prevention of ultraviolet B-induced wrinkle formation. J Invest Dermatol. 2001;117(3): 671-677.

21. Liu CJ, Tahara S, Gao S. Phosphorylation of extracellular signalregulated protein kinase in cultured keloid fibroblasts when stimulated by platelet-derived growth factor BB. Scand J Plast Reconstr Surg Hand Surg. 2003;37(6):321-324.

22. Yu W, Naim JO, Lanzafame RJ. The effect of laser irradiation on the release of bFGF from 3 T3 fibroblasts. Photochem Photobiol. 1994; 59(2):167-170.

23. Nolte SV, Xu W, Rennekampff HO, Rodemann HP. Diversity of fibroblasts - a review on implications for skin tissue engineering. Cells Tissues Organs. 2008;187(3):165-176.

24. Krejci NC, Cuono CB, Langdon RC, McGuire J. In vitro reconstitution of skin: fibroblasts facilitate keratinocyte growth and differentiation on acellular reticular dermis. J Dermatol. 1991;97(5):843-848.

International Journal of Nanomedicine

\section{Publish your work in this journal}

The International Journal of Nanomedicine is an international, peerreviewed journal focusing on the application of nanotechnology in diagnostics, therapeutics, and drug delivery systems throughout the biomedical field. This journal is indexed on PubMed Central, MedLine, CAS, SciSearch $\AA$, Current Contents ${ }^{\circledR} /$ Clinical Medicine,
25. Herson MR, Mathor MB, Altran S, Capelozzi VL, Ferreira MC. In vitro construction of a potential skin substitute through direct human keratinocyte plating onto decellularized glycerol-preserved allodermis. Artif Organs. 2001;25(11):901-906.

26. Filipe V, Hawe A, Jiskoot W. Critical evaluation of Nanoparticle Tracking Analysis (NTA) by NanoSight for the measurement of nanoparticles and protein aggregates. Pharm Res. 2010;27(5):796-810.

27. Dubey V, Mishra D, Asthana A, Jain NK. Transdermal delivery of a pineal hormone: melatonin via elastic liposomes. Biomaterials. 2006;27(18):3491-3496.

28. dos Santos T, Varela J, Lynch I, Salvati A, Dawson KA. Quantitative assessment of the comparative nanoparticle-uptake efficiency of a range of cell lines. Small. 2011;7(23):3341-3349.

29. Davies JE, Causton B, Bovell Y, Davy K, Sturt CS. The migration of osteoblasts over substrata of discrete surface charge. Biomaterials. 1986;7(3):231-233.

30. Hughes RC, Pena SD, Clark J, Dourmashkin RR. Molecular requirements for adhesion and spreading of hamster fibroblasts. Exp Cell Res. 1979; 121(2):307-314.

31. Kircheis R, Wightman L, Wagner E. Design and gene delivery activity of modified polyethylenimines. Adv Drug Deliv Rev. 2001;53(3): $341-358$.

32. Ito A, Matsuoka F, Honda H, Kobayashi T. Antitumor effects of combined therapy of recombinant heat shock protein 70 and hyperthermia using magnetic nanoparticles in an experimental subcutaneous murine melanoma. Cancer Immunol Immunother. 2004;53(1):26-32.

33. Chen T, Wong KF, Fenske DB, Palmer LR, Cullis PR. Fluorescentlabeled poly(ethylene glycol) lipid conjugates with distal cationic headgroups. Bioconjug Chem. 2000;11(3):433-437.

34. Palmer LR, Chen T, Lam AM, et al. Transfection properties of stabilized plasmid-lipid particles containing cationic PEG lipids. Biochim Biophys Acta. 2003;1611(1-2):204-216.

35. Fenske DB, Palmer LR, Chen T, Wong KF, Cullis PR. Cationic poly(ethyleneglycol) lipids incorporated into pre-formed vesicles enhance binding and uptake to BHK cells. Biochim Biophys Acta. 2001;1512(2):259-272.

36. Qaddoumi MG, Ueda H, Yang J, Davda J, Labhasetwar V, Lee VH. The characteristics and mechanisms of uptake of PLGA nanoparticles in rabbit conjunctival epithelial cell layers. Pharm Res. 2004;21(4): 641-648.

37. Chen $\mathrm{H}$, Chang $\mathrm{X}$, Weng $\mathrm{T}$, et al. A study of microemulsion systems for transdermal delivery of triptolide. J Control Release. 2004;98(3): $427-436$.

38. Kogan A, Garti N. Microemulsions as transdermal drug delivery vehicles. Adv Colloid Interface Sci. 2006;123-126:369-385.

39. Smyth HF Jr, Carpenter CP, Weil CS. The chronic oral toxicology of the polyethylene glycols. J Am Pharm Assoc Am Pharm Assoc (Baltim). 1955;44(1):27-30.

40. Huizing MT, Rosing H, Koopmans FP, Beijnen JH. Influence of Cremophor EL on the quantification of paclitaxel in plasma using highperformance liquid chromatography with solid-phase extraction as sample pretreatment. J Chromatogr B Biomed Sci Appl. 1998;709(1): $161-165$.

41. Bookstaff RC, Stuard SB, Ward SR, Pesik PK, Henwood SM. The safety of ethyl oleate is supported by a 91-day feeding study in rats. Regul Toxicol Pharmacol. 2004;39(2):202-213.

Journal Citation Reports/Science Edition, EMBase, Scopus and the Elsevier Bibliographic databases. The manuscript management system is completely online and includes a very quick and fair peer-review system, which is all easy to use. Visit http://www.dovepress.com/ testimonials.php to read real quotes from published authors. 